\title{
A Systematic Review and Meta-analysis of PD-1 and PD-L1 Inhibitors Monotherapy in Metastatic Gastric and Gastroesophageal Junction Adenocarcinoma
}

\author{
loannis A Voutsadakis ${ }^{1,2}$
}

\begin{abstract}
Immune checkpoint inhibitors are new targeted treatments that harness the body's immune system to attack cancers. Drugs that are most extensively used among checkpoint inhibitors inhibit the PD-L1 or PD-1 (programmed death 1) ligand or receptor pair and are currently approved for many cancer indications. In gastric or gastroesophageal junction adenocarcinomas one inhibitor, pembrolizumab has regulatory approval for PD-L1 positive carcinomas. This meta-analysis investigates available data on the efficacy of PD-L1 or PD-1 inhibitors as a class in gastric or gastroesophageal junction adenocarcinomas. The literature was reviewed to identify clinical studies that included arms with PD-L1 or PD-1 inhibitors as monotherapy in gastric or gastroesophageal junction adenocarcinomas. Relevant patient characteristics, outcomes, and adverse effects were recorded. Summary estimates of response rates (RR) and survival were calculated using a random or fixed effect model, depending on heterogeneity. Six studies with a total of 1068 patients were included in the analysis. The summary RR was $10.63 \%$ (95\% confidence interval (CI) $5.36-15.89 \%)$. The summary disease control rate (DCR) was $28.11 \%$ (95\% Cl $24.60-31.63 \%)$. Summary progression-free survival (PFS) was 1.59 months ( $95 \% \mathrm{Cl} 1.24-1.94$ months). Summary overall survival (OS) was 5.72 months ( $95 \% \mathrm{Cl} 0-12.19$ months). A subset of patients derived long-term benefits as seen in other cancer locations. The adverse effect rate was low and consistent with that in other disease locations. Low efficacy of immune checkpoint inhibitors as a class in gastric or gastroesophageal junction adenocarcinomas is observed in this analysis and stresses the need for effective biomarker use for the identification of most probable responders.
\end{abstract}

Keywords: Adenocarcinoma, Gastric cancer, Gastroesophageal junction, Immune checkpoint inhibitors.

Euroasian Journal of Hepato-Gastroenterology (2020): 10.5005/jp-journals-10018-1321

\section{INTRODUCTION}

Gastric and gastroesophageal junction adenocarcinomas represent one of the most common types of cancer and are more common in men than in women. ${ }^{1}$ Mortality from stomach cancer remains high and was 4.3 per 100,000 population in men and 2.3 per 100,000 in women in the US between 2011 and 2015. Some populations, such as, African Americans and Hispanics have even higher incidence and mortality rates. ${ }^{1}$ With an estimated 469.000 deaths in men and 254.000 deaths in women, gastric cancer rates the third cause of death by cancer in men and fifth in women worldwide. ${ }^{2}$

Immune blockade inhibitors (also called immune checkpoint inhibitors, ICls) are a new class of targeted anti-neoplastic drugs that block inhibitory receptors of the immune system and activate an immune response to the tumor. ${ }^{3}$ Currently, immune inhibitory receptors targeted in the clinic include CTLA-4 (cytotoxic T lymphocyte antigen 4) and PD-1 and its ligand PD-L1. Several other immune inhibitory receptor blockers are in advanced phase clinical studies. ${ }^{4}$ Monoclonal antibodies against CTLA-4 or PD-1 receptors and its ligand PD-L1 are approved for use in various malignancies, such as, lung cancer, melanoma, urothelial, renal, and hepatocellular carcinomas as well as Hodgkin's lymphoma. ${ }^{5-12}$ One of the immune checkpoint inhibitors, pembrolizumab has also obtained approval in the US for any tumor with microsatellite instability (MSI). ${ }^{13}$ Clinical trials leading to approval of ICls have been impressive in that they have documented long-lasting control of some previously refractory to chemotherapy patients and these drugs have now moved to the first-line metastatic setting in certain cancers. Studies in other cancers have produced less impressive results. Cancers responding to $\mathrm{ICls}$ tend to be those with a higher
${ }^{1}$ Department of Medical Oncology, Sault Area Hospital, Sault Ste Marie, Ontario, Canada

${ }^{2}$ Division of Clinical Sciences, Section of Internal Medicine, Northern Ontario School of Medicine, Sudbury, Ontario, Canada

Corresponding Author: loannis A Voutsadakis, Department of Medical Oncology, Sault Area Hospital, Sault Ste Marie, Ontario, Canada, Phone: +1 7057593434, e-mail: ivoutsadakis@yahoo.com

How to cite this article: Voutsadakis IA. A Systematic Review and Metaanalysis of PD-1 and PD-L1 Inhibitors Monotherapy in Metastatic Gastric and Gastroesophageal Junction Adenocarcinoma. Euroasian J HepatoGastroenterol 2020;10(2):56-63.

Source of support: Nil

Conflict of interest: None

tumor mutation burden, while non-responsive cancers tend to have a lower mutation burden. ${ }^{14,15}$ Besides the mutation burden, other possible predictive markers of response to ICls include MSI and tumor micro-environment PD-L1 expression (for PD-1 inhibitors). Both these markers, in contrast to tumor burden, are on some occasions embedded in the indication of the drugs. ${ }^{6,13}$

Immune blockade inhibitors have been studied in clinical trials for many common gastrointestinal cancers. ${ }^{16}$ Gastric and gastroesophageal junction adenocarcinomas constitute one of the gastrointestinal cancer groups where ICls have been investigated. Results of these trials have been encouraging in subsets of patients and regulatory approval has been granted by the USFDA to the PD-L1 inhibitor pembrolizumab for gastric gastroesophageal junction adenocarcinomas that express PD-L1. ${ }^{17}$ The current investigation

(C) The Author(s). 2020 Open Access This article is distributed under the terms of the Creative Commons Attribution 4.0 International License (https://creativecommons. org/licenses/by-nc/4.0/), which permits unrestricted use, distribution, and non-commercial reproduction in any medium, provided you give appropriate credit to the original author(s) and the source, provide a link to the Creative Commons license, and indicate if changes were made. The Creative Commons Public Domain Dedication waiver (http://creativecommons.org/publicdomain/zero/1.0/) applies to the data made available in this article, unless otherwise stated. 
presents a meta-analysis of trials that included arms with PD-1 or PD-L1 monotherapy to inform on the efficacy of these drugs in gastric and gastroesophageal adenocarcinomas. It will also seek to analyze any data on markers predictive of response arising from these trials.

\section{Methods}

A meta-analysis of all phase II and III studies that included monotherapy arms of PD-1 and PD-L1 inhibitors nivolumab, pembrolizumab, atezolizumab, avelumab, and durvalumab in metastatic gastric or gastroesophageal adenocarcinoma patients was performed to better understand the efficacy of these drugs with a similar mechanism of action in these cancers. These five checkpoint inhibitors are currently in clinical use for different indications in oncology.

The search of the literature for this meta-analysis was performed in the Medline or PubMed database (www.ncbi.nlm. nih.gov/pubmed), the EMBASE (Excerpta Medica) database, and the Cochrane Central Register of Controlled Trials with the search terms "nivolumab" or "pembrolizumab" or "atezolizumab" or "avelumab" or "durvalumab" and "gastric cancer" or "gastroesophageal cancer". The date of the last search of the databases was November 20, 2019. Studies of any prospective design (except for dose-finding phase I) were included if they were in the English language and if they included treatment arms that received therapy with one of the checkpoint inhibitors as monotherapy. In contrast, articles in other languages, case reports or small (less than 15 patients) nonrandomized-case series, pre-clinical studies or reviews and opinion articles were excluded. Also excluded were studies of combination therapies of checkpoint inhibitors with other checkpoint inhibitors, other targeted drugs or chemotherapeutics. References of retrieved articles were searched manually for additional studies of relevance.

Studies retained were scanned for data describing the demographics of the treated population, characteristics of the treated diseases and the efficacy and toxicity of treatment of interest. Population data of patients in the arms treated with one of the five checkpoint inhibitors of interest extracted for the analysis included age of the patients, ECOG performance status, number and type of previous lines of treatment for metastatic disease, and histologic sub-type. Additional data of interest that were searched and recorded when available included positivity for HER2, PD-L1, EBV, presence of MSI, and tumor mutation burden. Efficacy outcomes of interest included RR, DCR, median OS, and median PFS, with their respective $95 \% \mathrm{Cl}$. Information on overall and grade 3 and 4 toxicity rates were also extracted from included studies. All studies of the meta-analysis were evaluated for the risk of bias with the revised Cochrane risk-of-bias for randomized trials (RoB 2.0) tool. ${ }^{18}$

Descriptive statistics were calculated for all patients' characteristics of interest and outcome measures. Pooled outcomes rates were weighted according to the number of patients in each series. Heterogeneity among the studies was evaluated with Cochran's $Q$ and $I^{2}$ tests. The fixed or random-effect model was used if heterogeneity was low or high, respectively. ${ }^{19}$ Calculations were performed in Excel (Microsoft Corp.) as previously described with some modifications ${ }^{20}$ and re-performed for confirmation of results with Open Meta-analyst, an open-source online software, developed by researchers at Brown University (www.cebm.brown. edu/openmeta/). ${ }^{21}$ The two tools produced similar results.

No funding was received from any source for the performance of this systematic review and meta-analysis. No additional protocol exists for this study.

\section{Results}

Literature search disclosed 46 publications on nivolumab, 53 publications on pembrolizumab, 8 publications on atezolizumab, 10 publications on avelumab, and 6 publications on durvalumab in gastric or gastroesophageal adenocarcinoma (Fig. 1). Among these publications, a total of 115 articles were reviews, opinions, or clinical studies that did not include arms with PD-1 or PD-L1 inhibitors as monotherapy and thus were excluded. One study of nivolumab was excluded because it concerned squamous histology. ${ }^{22}$ Another small study of 23 patients treated with nivolumab included mostly squamous carcinomas and only five patients with adenocarcinomas and was also excluded. ${ }^{23}$ Clinical studies that fulfilled the inclusion criteria included three publications on pembrolizumab, ${ }^{24-26}$ two articles on nivolumab, ${ }^{27,28}$ and one article with avelumab. ${ }^{29}$ No trials concerning gastric or gastroesophageal adenocarcinoma with monotherapy arms of atezolizumab or durvalumab were identified. The six articles included were published between 2016 and 2018 and concerned the monotherapy arm of multi-arm trials $(n=4)$ or single-arm reports $(n=2)$ (Table 1). The six studies included a total of 1068 patients in their PD-L1 or PD-1 inhibitors monotherapy arms that were analyzed for efficacy and toxicity outcomes. Five of the six trials were performed globally and one ${ }^{28}$ was performed only in far Eastern countries (Table 1). One study included patients in the second line of treatment, ${ }^{24}$ three studies included patients in the second line or higher line of treatment ${ }^{25,27,29}$ and two studies included patients in the third line or higher of metastatic treatment. ${ }^{26,28}$ The dose of medication in the three pembrolizumab studies was a fixed dose of $200 \mathrm{mg}$ every 3 weeks in two studies and $10 \mathrm{mg} / \mathrm{kg}$ every 2 weeks in the third. Both nivolumab studies used a dose of $3 \mathrm{mg} / \mathrm{kg}$ every 2 weeks. The avelumab trial used a dose of $10 \mathrm{mg} / \mathrm{kg}$ every 2 weeks.

The median age of the patients in the six studies ranged between 59 and 64 years. Most patients (62.3\%) had an ECOG PS of 1 and the rest (37.6\%) had an ECOG PS of 0 (Table 2). The number of prior lines of therapy in the metastatic setting was 1 in 239 patients (22.4\%), 2 lines in 385 patients (36.1\%) and 3 or more lines of therapy in 437

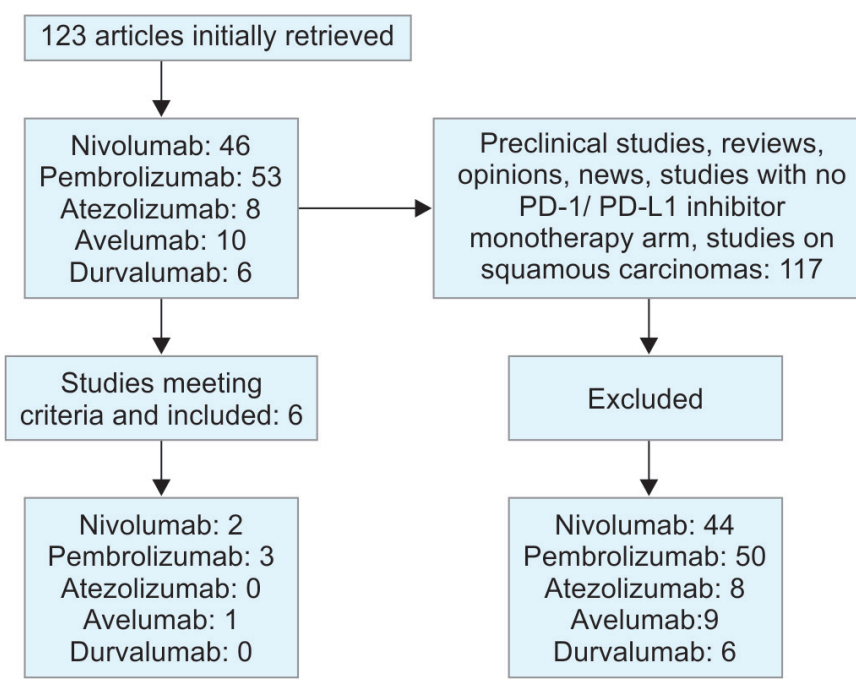

Fig. 1: Flow diagram of the studies evaluated for the meta-analysis and reasons for exclusion 
PD-1 and PD-L1 in Gastroesophageal Cancer

Table 1: The six studies included in the current meta-analysis

\begin{tabular}{|c|c|c|c|c|c|c|c|c|}
\hline $\begin{array}{l}\text { First author } \\
\text { [Reference] }\end{array}$ & $\begin{array}{l}\text { Study registration } \\
\text { number }\end{array}$ & $\begin{array}{l}\text { Year of } \\
\text { publication }\end{array}$ & Country & $\begin{array}{l}\text { Total number of } \\
\text { patients }\end{array}$ & $\begin{array}{l}\text { Number of patients } \\
\text { in analyzed arm }\end{array}$ & $\begin{array}{l}\text { Line of } \\
\text { treatment }\end{array}$ & $R R(\%)$ & $D C R(\%)$ \\
\hline Janjigian $^{27}$ & NCT01928394 & 2018 & $\begin{array}{l}\text { USA, Europe } \\
\text { (5 countries) }\end{array}$ & 160 & 59 & $\geq 2 \mathrm{nd}$ & 11.86 & 32.2 \\
\hline Shitara ${ }^{24}$ & NCT02370498 & 2018 & 30 countries & 395 & 196 & 2nd & 15.82 & NA \\
\hline Muro $^{25}$ & NCT01848834 & 2016 & $\begin{array}{l}\text { USA, Israel, Japan, } \\
\text { Korea, Taiwan }\end{array}$ & 39 & 39 & $\geq 2$ nd & 20.51 & 33.33 \\
\hline Fuchs $^{26}$ & NCT02335411 & 2018 & 16 countries & 259 & 259 & $\geq 3 \mathrm{rd}$ & 11.58 & 27.03 \\
\hline Kang $^{28}$ & NCT02267343 & 2017 & $\begin{array}{l}\text { Japan, Korea, } \\
\text { Taiwan }\end{array}$ & 493 & 330 & $\geq 3$ rd & 9.09 & 32.73 \\
\hline Bang $^{29}$ & NCT02625623 & 2018 & Global & 371 & 185 & 2nd, 3rd & 2.16 & 22.16 \\
\hline
\end{tabular}

Table 2: Characteristics of the included patients from the six studies. Some characteristics were available in only part of the studies. The intestinal histologic type includes variants

\begin{tabular}{|c|c|c|}
\hline & Patients $(n=1068)$ & $\%$ \\
\hline Age medians (range) & $59-64(24-89)$ & $56(22-85)$ \\
\hline \multicolumn{3}{|l|}{ ECOG PS } \\
\hline 0 & 402 & 37.6 \\
\hline 1 & 665 & 62.3 \\
\hline 2 & 1 & 0.1 \\
\hline Histologic type & $n=970$ (4 studies) & \\
\hline Intestinal & 520 & 53.6 \\
\hline Diffuse/mixed & 174 & 17.9 \\
\hline Unknown/other & 276 & 28.5 \\
\hline \multicolumn{3}{|l|}{ Prior lines of chemotherapy } \\
\hline 0 & 6 & 0.6 \\
\hline 1 & 239 & 22.4 \\
\hline 2 & 385 & 36.1 \\
\hline$\geq 3$ & 437 & 40.9 \\
\hline Location & $n=737$ (5 studies) & \\
\hline GEJ & 309 & 41.9 \\
\hline Stomach & 428 & 58.1 \\
\hline PD-L1 & $n=821$ & \\
\hline Positive & 461 & 56.2 \\
\hline Negative & 360 & 43.8 \\
\hline MSI status & $\begin{array}{l}n=199 \text { (some patients } \\
\text { from } 2 \text { studies) }\end{array}$ & \\
\hline MSI-H & 14 & 7.0 \\
\hline MSI-L & 185 & 93.0 \\
\hline HER2 status & $n=331$ ( 2 studies) & \\
\hline Positive & 107 & 32.3 \\
\hline Negative & 224 & 67.7 \\
\hline \multicolumn{3}{|l|}{ Efficacy } \\
\hline Median OS (months) $(95 \% \mathrm{Cl})$ & 5.72 & $0-12.19$ \\
\hline Median PFS (months) $(95 \% \mathrm{Cl})$ & 1.59 & $1.24-1.94$ \\
\hline $\mathrm{RR} \%(95 \% \mathrm{Cl})$ & 10.63 & $5.36-15.89$ \\
\hline DCR\% (95\% Cl) & 28.11 & $24.6-31.63$ \\
\hline
\end{tabular}

patients (40.9\%). Information on the location of the primary tumor was available in 737 patients from 5 studies. The gastroesophageal junction was the location of the primary tumor in $41.9 \%$ of those patients and the rest of the stomach in $58.1 \%$ of the patients (Table 2).
The majority of the patients (53.6\%) had an intestinal histologic type and $17.9 \%$ of patients had a diffuse or mixed histologic type. Regarding PD-L1 and HER2 status, the information was available in 821 and 331 patients, respectively. A total of $56.2 \%$ of patients were positive for PD-L1 and $32.3 \%$ of patients were positive for HER2. Microsatellite status (MSI) was reported in two studies, ${ }^{24,25}$ although these data were not available in all patients even in these two studies. Overall 199 patients had data for MSI status and 14 patients (7\%) were MSI-H (MSI High). No studies included data on tumor mutation burden or EBV status of the tumors. Similarly, no data were presented in any of the six studies regarding smoking which may be related to the mutation burden of the tumors.

The risk of bias is estimated to be moderate in five of the six studies due to their phase II non-randomized design or randomized open-label design. ${ }^{24-27,29}$ These designs could be associated with biases in the domains of intervention assignment and adhesion and of outcomes measurement. One study was double-blind, placebocontrolled. ${ }^{28}$ This design has a lower risk of bias.

The analysis of RR from the six studies disclosed a summary RR of $10.63 \%$ (95\% Cl 5.36-15.89\%) (Fig. 2). Evaluation of heterogeneity between studies confirmed a high level of heterogeneity with an $I^{2}$ value of 87 (Cochran's $Q=40.23, x^{2}$ $p<0.0001)$ and thus a random effect model was used for the meta-analysis calculations.

Disease control rate was not available in one study ${ }^{22}$ and the remaining five studies that included a total of 872 patients formed the basis of DCR calculation. Analysis for DCR showed that the five studies had low heterogeneity for this outcome with an $I^{2}$ value of 1.7 (Cochran's $Q=5.84, x^{2} p=0.21$ ). As a result, the fixedeffect model was retained. The summary DCR was $28.11 \%(95 \% \mathrm{Cl}$ 24.60-31.63\%) (Fig. 3).

Progression-free survival (PFS) analysis was performed under the random effect model as the level of heterogeneity between studies was high $\left(I^{2}=98\right.$, Cochran's $\left.Q=292, x^{2} p<0.00001\right)$. Summary PFS was 1.59 months (95\% Cl 1.24-1.94 months) (Fig. 4).

Finally, the summary OS was 5.72 months $(95 \% \mathrm{Cl} 0-12.19$ months) (Fig. 5). This analysis was also performed under the random effect model as the level of heterogeneity between studies was high $\left(I^{2}=66\right.$, Cochran's $\left.Q=14.92, x^{2} p=0.01\right)$.

Among the five studies that included both PD-L1 positive and PD-L1 negative patients, two studies reported RR separately for the two PD-L1 groups ${ }^{26,27}$ and two other studies reported a median OS separately. ${ }^{28,29}$ In one study that reported on RRs, RR in PD-L1positive patients was $13 \%$ and RR in PD-L1-negative patients was $4 \% .{ }^{27}$ In the other study, RR was $15.5 \%$ in PD-L1-positive patients 


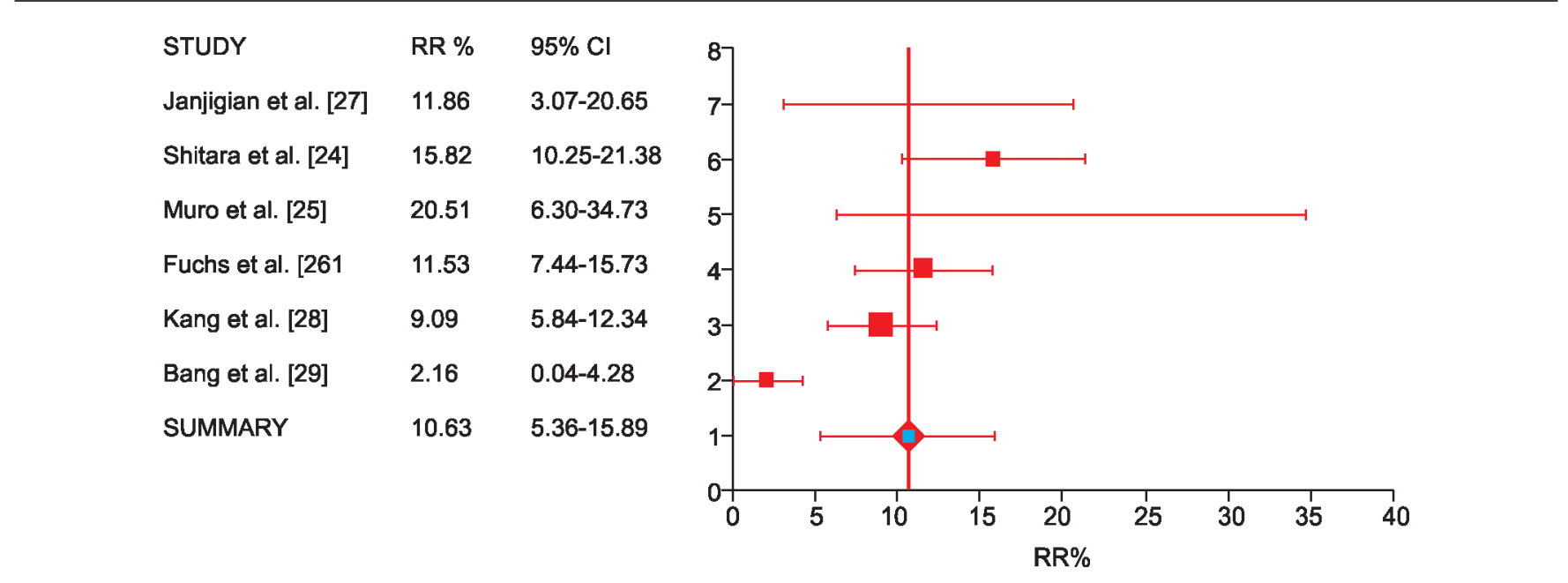

Fig. 2: Diagram of RR and 95\% Cl of studies of PD-1 and PD-L1 inhibitors in metastatic gastric and gastroesophageal junction adenocarcinoma. The estimated overall RR was $10.63 \%$ (95\% Cl 5.36-15.89\%)

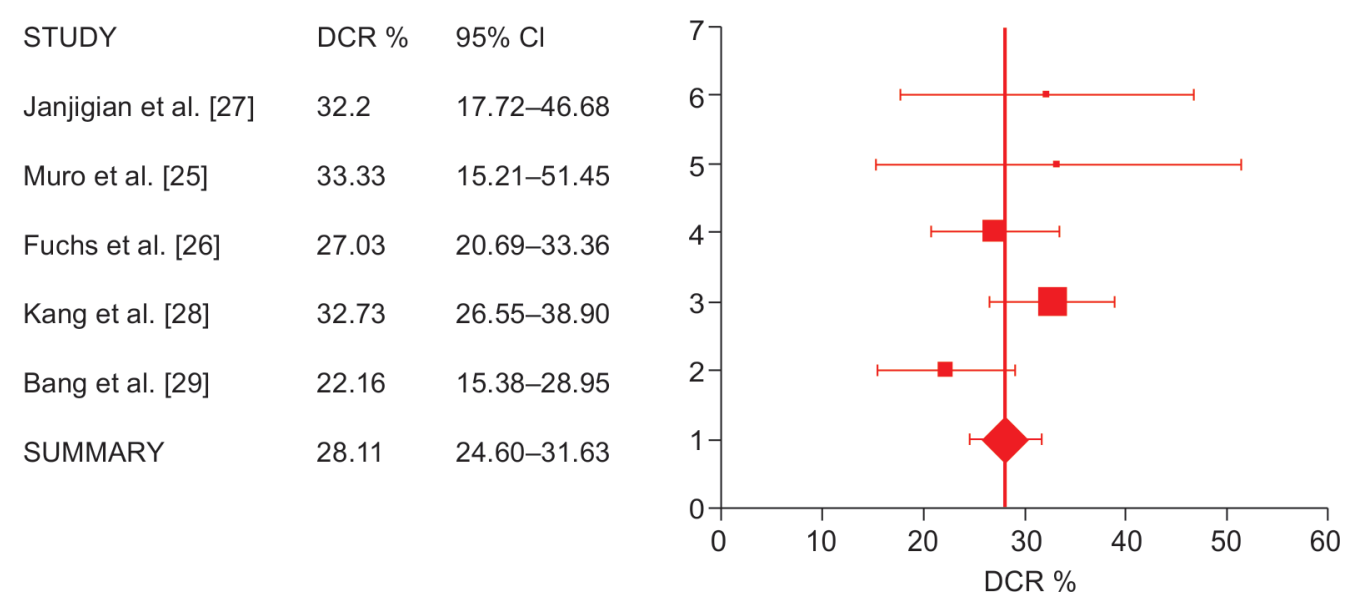

Fig. 3: Diagram of the meta-analysis of DCR and $95 \% \mathrm{Cl}$. Five studies were included in this analysis. The overall DCR was $28.11 \%(95 \% \mathrm{Cl} 24.60-31.63 \%)$

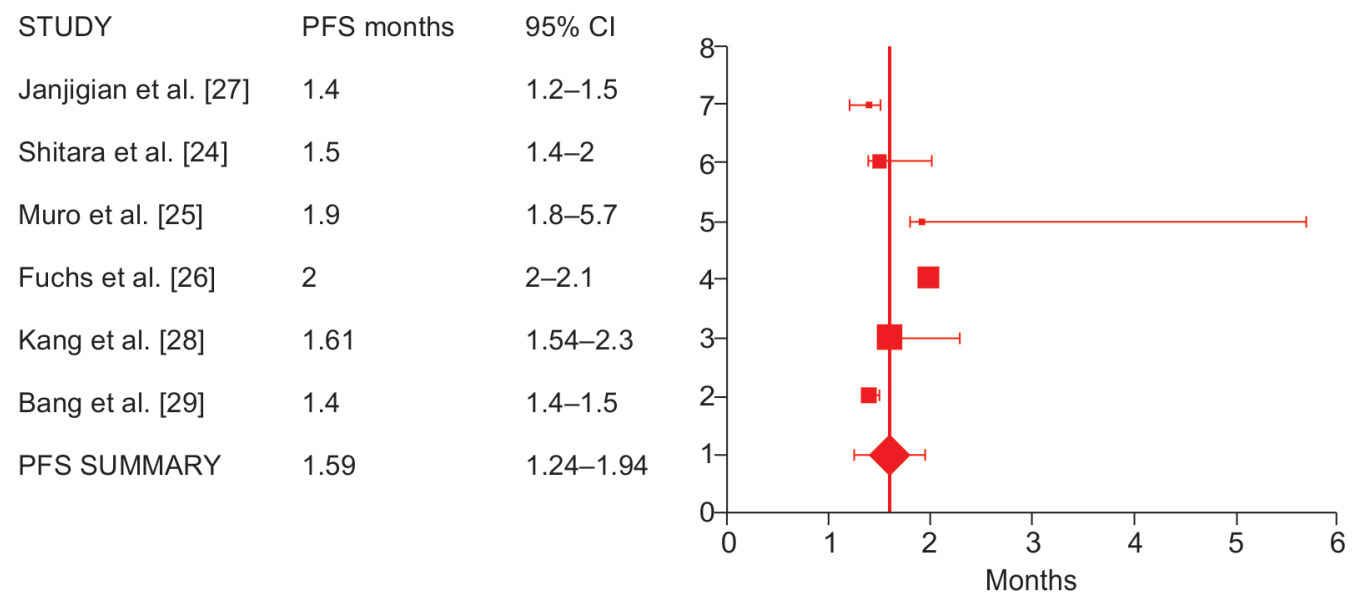

Fig. 4: Diagram of PFS in the six studies that form the basis of the report and summary estimates of PFS. Summary PFS was 1.59 months (95\% Cl 1.24-1.94 months) 


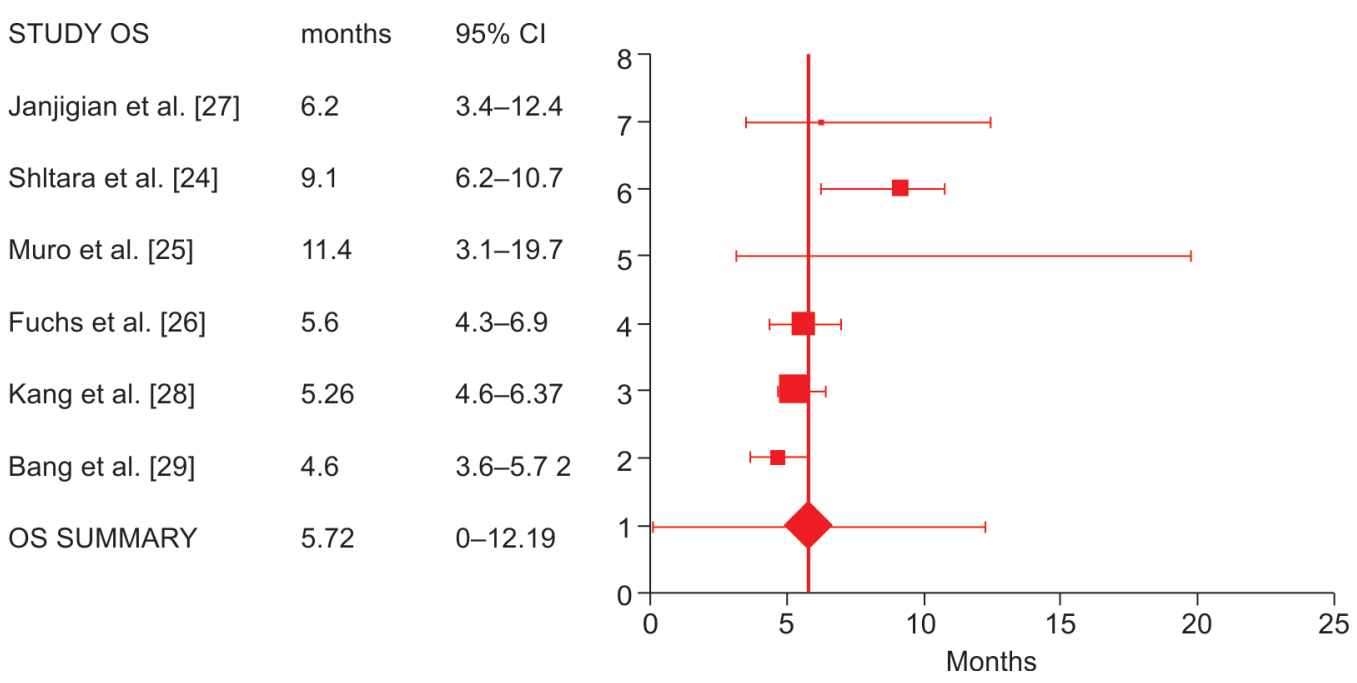

Fig. 5: Meta-analysis of OS and $95 \% \mathrm{Cl}$. Summary OS was 5.72 months (95\% Cl 0-12.19 months)

and 6.4\% in PD-L1-negative patients. ${ }^{26}$ In contrast, in both studies that reported on the median OS, these were no better in PD-L1 positive patients (median OS 5.22 months and 4 months in PD-L1 positive patients and 6.05 months and 4.6 months in PD-L1 negative patients, respectively).

Response rates of the trial that included only patients at the second line of treatment $(15.82 \%, 95 \% \mathrm{Cl}: 10.25-21.38 \%)^{24}$ was only minimally higher than the RR of the two trials that included only patients at the third or later lines of treatment $(11.58 \%, 95 \%$ Cl: $7.44-15.73 \%$ and $9.09 \%, 95 \% \mathrm{Cl}: 5.84-12.34 \%$, respectively). ${ }^{26,28}$ Similarly, PFS was not significantly different (1.5 months in the study with second-line patients, 2 months and 1.61 months in the two studies with a third and higher line of treatment patients). Overall survival was somewhat higher in the second-line study (9.1 months) than in the two studies that included third and higher line patients (5.6 and 5.26 months, respectively) but with overlapping $95 \% \mathrm{Cls}$.

Treatment with PD-1 and PD-L1 inhibitors was very well tolerated and the only adverse effects of all grades that were observed in more than $10 \%$ of patients were asthenia and pruritus or cutaneous rashes (Table 3). Severe immune-related adverse effects were very rare.

\section{Discussion}

Metastatic gastric and gastroesophageal adenocarcinoma remains a lethal disease despite increasing options for treatment. ${ }^{30}$ Immunotherapy with PD-L1 and PD-1 inhibitors provides an additional option of treatment. These drugs are monoclonal antibodies that inhibit the interaction of the inhibitory PD-L1 or PD-1 pair and thus allow activation of effector cytotoxic T cells and NK cells to attack tumor cells if appropriate neo-antigens are presented on the tumor cells' surface. ${ }^{31,32}$ Tumor types with high mutation loads such as lung cancers and melanoma as well as tumors with MSI independently of the primary site have been found to be most sensitive to PD-L1 or PD-1 inhibition.

In the current report six studies that included arms with patients with metastatic gastric or gastroesophageal adenocarcinomas who were treated with a PD-L1 or PD-1 inhibitor as monotherapy were identified and analyzed. A brief discussion of the design and results of the six studies follows.
Table 3: All-grade and grade 3-4 toxicities observed in the six included studies. Some toxicities were included in the toxicity discussion of some studies but not in others and thus the denominator is less than a total of 1068 patients

\begin{tabular}{|c|c|c|c|c|}
\hline Toxicity \% & $\begin{array}{l}\text { All grades or } \\
\text { total number } \\
\text { of toxicity } \\
\text { information } \\
\text { mentioned }\end{array}$ & $\begin{array}{l}\text { All } \\
\text { grades } \\
\text { (\%) }\end{array}$ & $\begin{array}{l}\text { Grade } 3-4 \text { or } \\
\text { total number } \\
\text { of toxicity } \\
\text { information } \\
\text { available }\end{array}$ & $\begin{array}{l}\text { Grade } \\
3-4 \\
(\%) \\
\end{array}$ \\
\hline Asthenia/fatigue & $147 / 1068$ & 13.8 & $18 / 1068$ & 1.7 \\
\hline Neutropenia & 0 & - & 0 & - \\
\hline $\begin{array}{l}\text { Febrile } \\
\text { neutropenia }\end{array}$ & 0 & - & 0 & - \\
\hline Anemia & $29 / 640$ & 4.5 & $14 / 640$ & 2.2 \\
\hline $\begin{array}{l}\text { Peripheral } \\
\text { neuropathy }\end{array}$ & $4 / 235$ & 1.7 & 0 & - \\
\hline Nausea & $69 / 970$ & 7.1 & $3 / 970$ & 0.3 \\
\hline Diarrhea & $76 / 1029$ & 7.4 & $8 / 1029$ & 0.8 \\
\hline Alopecia & $1 / 381$ & 0.3 & - & - \\
\hline Pruritus/rash & $115 / 883$ & 13.0 & $2 / 883$ & 0.2 \\
\hline \multicolumn{5}{|l|}{ Immune related } \\
\hline Hyperthyroidism & $12 / 526$ & 2.3 & 0 & - \\
\hline Hypothyroidism & $59 / 824$ & 7.2 & $1 / 824$ & 0.1 \\
\hline $\begin{array}{l}\text { Interstitial lung } \\
\text { disease }\end{array}$ & $5 / 565$ & 0.9 & - & - \\
\hline Colitis & $2 / 526$ & 0.4 & - & - \\
\hline Hypophysitis & $3 / 526$ & 0.6 & - & - \\
\hline $\begin{array}{l}\text { Hepatitis/ } \\
\text { enzymes } \\
\text { elevation }\end{array}$ & $47 / 574$ & 8.2 & $21 / 574$ & 3.6 \\
\hline
\end{tabular}

The first nivolumab study included was a randomized phase II trial with three arms in chemotherapy pretreated patients without selection for PD-L1 status. ${ }^{27}$ Besides the nivolumab monotherapy arm, the trial included two arms with combinations 
of nivolumab and the CTLA-4 inhibitor ipilimumab in different doses. Objective RR (the primary endpoint) were $12 \%$ with nivolumab monotherapy, $24 \%$ in the arm that received nivolumab $1 \mathrm{mg} / \mathrm{kg}$ and ipilimumab $3 \mathrm{mg} / \mathrm{kg}$ and $8 \%$ in the arm that received nivolumab $3 \mathrm{mg} / \mathrm{kg}$ and ipilimumab $1 \mathrm{mg} / \mathrm{kg}$ with overlapping $95 \%$ confidence intervals in the three arms. The second nivolumab study included in the current analysis was a randomized phase III trial of nivolumab vs a placebo arm in the third or later line of treatment of metastatic gastric and gastroesophageal junction cancer also without selection for PD-L1 status. ${ }^{28}$ The 1-year OS was $26.6 \%$ in the nivolumab arm vs $10.9 \%$ with placebo. The study showed a modest but statistically significant median OS increase with nivolumab from 4.14 months in the placebo arm to 5.32 months in the nivolumab arm.

Three studies included in the current analysis concerned pembrolizumab. A phase Ib study allowed patients with both PD-L1-positive and PD-L1-negative metastatic gastric and gastroesophageal junction adenocarcinoma. ${ }^{25}$ This study observed partial responses in eight of the 36 (22\%) evaluable patients and a median OS of 11.4 months. Importantly, some patients remained on treatment for protracted time periods exceeding 6 months. ${ }^{25}$ A second pembrolizumab trial was an extensive phase II study that included 259 patients with gastroesophageal cancer, both PD-L1-positive and PD-L1-negative, who were treated in the third line metastatic setting. ${ }^{26}$ Response rate was $11.58 \%$ and stable disease was observed in $15.45 \%$ of patients, for a CBR of $27.03 \%$. The third pembrolizumab study included in the current analysis was a randomized, open-label, multicenter phase III trial in pretreated patients. ${ }^{24}$ It included only PD-L1-positive patients defined as $1 \%$ or more positive staining in histologic sections counting both cancer and inflammatory cells. The second arm in this trial received weekly paclitaxel. Although better tolerated, pembrolizumab did not improve PFS or OS compared with paclitaxel. ${ }^{24}$

The anti-PD-L1 antibody avelumab was the subject of investigation in the last included study which was a phase III trial in patients with metastatic gastric or gastroesophageal junction adenocarcinoma irrespective of PD-L1 status in the second or third-line setting. ${ }^{29}$ This study compared avelumab with a control arm of physician's choice chemotherapy. Most patients (64.5\%) in the control arm received irinotecan and $29 \%$ received paclitaxel. The trial showed no improvement in OS or PFS with avelumab vs chemotherapy. ${ }^{29}$

The pooled analysis of the six monotherapy arms disclosed a low RR of about $10 \%$ and an equally unimpressive DCR of $28.1 \%$. Summary PFS was just above 1.5 months and summary OS was below 6 months. These results confirm a low efficacy of PD-L1 or PD-1 inhibitors in mostly unselected patients with metastatic adenocarcinoma of the stomach and gastroesophageal junction. Similar results have been reported in a phase II study of nivolumab in 64 patients with esophageal squamous cell carcinoma. ${ }^{22}$ Objective RR in this study was $17 \%$ and DCR was $42 \%$. Despite these mediocre results several points deserve noticing regarding the efficacy of PD-L1 or PD-1 inhibitors in gastric and gastroesophageal junction adenocarcinoma. First as seen in most other cancers there were long-term responses among responders and in fact, long-term responses are not rare. As a result, a significant minority of patients derive long-term benefits. Thus, the challenge becomes to identify biomarkers that would predict the efficacy of these immunotherapeutic drugs. In this direction, the first identified marker that has been intuitively identified is the expression of the target ligand, PD-L1. The expression of PD-L1 has been tested in other cancers and is successfully used as a biomarker for patient selection for treatment with pembrolizumab in lung cancer. ${ }^{6}$ In contrast, it is not required for response in other cancers and other PD-1 or PD-L1 inhibitors. The studies in gastric or gastroesophageal junction adenocarcinomas, besides one study ${ }^{24}$, allowed for the inclusion of patients independently from their PD-L1 status. Sub-group analysis of RR in two trials showed a numerically higher rate in PD-L1 positive patients than in PD-L1 negative counterparts (13 vs $4 \%$ in one trial and 15.5 vs $6.4 \%$ in the other). ${ }^{26,27}$ However, two other trials that reported median OS separately in PD-L1 positive and negative patients disclosed no differences. ${ }^{28,29}$ Another prognostic marker for immune blockade inhibitors efficacy is MSI status. A comparatively small number of patients from two studies (total of 174 patients) had a known MSI status. Among those, seven patients were MSI-H and four of them (57.1\%) showed a response. In contrast, only 15 out of 167 patients with MSI-L status (9.0\%) had a response to treatment. Other prognostic factors for Immune blockade inhibitors efficacy include tumor mutation burden and EBV status. ${ }^{14,33}$ Unfortunately, no data on these factors have been reported in any of the six studies. Similarly, no information was provided in any of the studies regarding responses in histologic subtypes (intestinal vs diffuse) or HER2-positive vs negative tumors.

Line of treatment may affect the observed efficacy of a given therapy in the metastatic setting, as treatments tend to become less efficacious as a given cancer progresses along previous lines of therapy developing the most complex genetic aberrations. Three of the studies of the current meta-analysis included patients across lines of metastatic treatment and thus are not informative regarding this point. ${ }^{25,27,29}$ Among the other three trials, one included only second-line patients ${ }^{24}$ and two included third or higher-line patients. ${ }^{26,28}$ Comparison of survival of patients that received PD-1 or PD-L1 inhibitors in the different lines of therapy shows that PFS was very similar in the second and third or higher lines of treatment but OS was numerically longer in the second line of therapy. Of note, however, the study that included only patients in the second line was also the only one that had PD-L1 positivity as an inclusion criterion. ${ }^{24}$

Adverse effects profile of PD-L1 or PD-1 inhibitors in gastric or gastroesophageal junction adenocarcinomas were entirely consistent with this profile reported in other cancers. A metaanalysis of 31 studies of nivolumab across different cancers, for example, reported a rate of $10.4 \%$ for asthenia of all grades, $13 \%$ for the rash of all grades, $11.8 \%$ for nausea of all grades, and $12.1 \%$ for diarrhea of all grades. ${ }^{34}$ These were all similar to adverse effects observed in the current meta-analysis (Table 3). Regarding immune-related adverse effects, the same meta-analysis of 31 studies observed hypothyroidism and transaminases elevations as the most common immune-related adverse effects and also very consistent with the current meta-analysis.

Limitations of the present meta-analysis consist mostly of the aforementioned lack of information regarding predictive markers that could enrich for responding patients and guide further development of the drugs. Another limitation could stem from the inclusion of studies with both anti-PD-1 drugs (nivolumab and pembrolizumab) and anti-PD-L1 drugs (avelumab) in the analysis. Despite blocking the same pair of ligand or receptor, the target proteins expression defers in the various cells of the tumor micro-environment and thus therapeutic effects may not 
be completely super-imposable. Nevertheless, results with the only study with an anti-PD-L1 agent available and included in the analysis were generally consistent with the results of the remaining five studies of anti-PD-1 drugs. However, one notices that efficacy measures in the avelumab study tended to be the lowest among studies.

The current systematic review and meta-analysis observed low RR and survival outcomes of PD-1 or PD-L1 inhibitors in mostly unselected patients with gastric or gastroesophageal junction adenocarcinomas. Further establishment of this class of drugs in this disease will rely on marker-identified patients with higher response probabilities as well as on synergistic combinations. Predictive markers, such as, MSI status, EBV status, tumor mutation burden, as well as PD-L1 expression already exist and await further refinement for clinical use. As mentioned previously the only current approval of a PD-1 or PD-L1 inhibitor by the FDA in gastric or gastroesophageal junction adenocarcinomas is for pembrolizumab for PD-L1 positive cancers. ${ }^{17}$ Improvement of these markers, for example taking into consideration specific mutation signatures with the differing propensity for neo-antigen production, could provide even more predictive power. Further studies results are eagerly awaited. ${ }^{35,36}$

\section{References}

1. Siegel RL, Miller KD, Jemal A. Cancer statistics, 2018. CA Cancer J Clin 2018;68(1):7-30. DOI: 10.3322/caac.21442.

2. Torre LA, Bray F, Siegel RL, et al. Global cancer statistics, 2012. CA Cancer J Clin 2015;65(2):87-108. DOI: 10.3322/caac.21262.

3. Voutsadakis IA. Immune blockade inhibition in breast cancer. Anticancer Res 2016;36(11):5607-5622. DOI: 10.21873/anticanres.11145.

4. Advani R, Flinn I, Popplewell L, et al. CD47 blockade by Hu5F9G4 and rituximab in non-Hodgkin's lymphoma. N Engl J Med 2018;379(18):1711-1721. DOI: 10.1056/NEJMoa1807315.

5. Borghaei $\mathrm{H}$, Paz-Ares L, Horn L, et al. Nivolumab versus docetaxel in advanced nonsquamous non-small-cell lung cancer. N Engl J Med 2015;373(17):1627-1639. DOI: 10.1056/NEJMoa1507643.

6. Garon EB, Rizvi NA, Hui R, et al. Pembrolizumab for the treatment of non-small-cell lung cancer. N Engl J Med 2015;372(21):2018-2028. DOI: 10.1056/NEJMoa1501824.

7. Brahmer J, Reckamp KL, Baas P, et al. Nivolumab versus docetaxel in advanced squamous-cell non-small-cell lung cancer. N Engl J Med 2015;373(2):123-135. DOI: 10.1056/NEJMoa1504627.

8. Robert C, Long GV, Brady B, et al. Nivolumab in previously untreated melanoma without BRAF mutation. N Engl J Med 2015;372(4):320330. DOI: 10.1056/NEJMoa1412082.

9. Motzer RJ, Escudier B, McDermott DF, et al. Nivolumab versus everolimus in advanced renal-cell carcinoma. N Engl J Med 2015;373(19):1803-1813. DOI: 10.1056/NEJMoa1510665.

10. Powles T, Eder JP, Fine GD, et al. MPDL3280A (anti-PD-L1) treatment leads to clinical activity in metastatic bladder cancer. Nature 2014;515(7528):558-562. DOI: 10.1038/nature13904.

11. El-Khoueiry AB, Sangro B, Yau T, et al. Nivolumab in patients with advanced hepatocellular carcinoma (CheckMate 040): an openlabel, non-comparative, phase 1/2 dose escalation and expansion trial. Lancet 2017;389(10088):2492-2502. DOI: 10.1016/S01406736(17)31046-2.

12. Chen R, Zinzani PL, Fanale MA, et al. Phase Il study of the efficacy and safety of pembrolizumab for relapsed/refractory classic Hodgkin lymphoma. J Clin Oncol 2017;35(19):2125-2132. DOI: 10.1200/ JCO.2016.72.1316.

13. Lemery S, Keegan P, Pazdur R. First FDA approval agnostic of cancer site - when a biomarker defines the indication. N Engl J Med 2017;377(15):1409-1412. DOI: 10.1056/NEJMp1709968.

14. Kandoth C, McLellan MD, Vandin F, et al. Mutational landscape and significance across 12 major cancer types. Nature 2013;502(7471):333339. DOI: $10.1038 /$ nature12634.
15. Vogelstein B, Papadopoulos N, Velculescu VE, et al. Cancer genome landscapes. Science 2013;339(6127):1546-1558. DOI: 10.1126/ science.1235122.

16. Voutsadakis IA. Immune blockade inhibitors and the radiation abscopal effect in gastrointestinal cancers. World J Gastrointest Oncol 2018;10(9):221-227. DOI: 10.4251/wjgo.v10.i9.221.

17. Fashouin-Aje L, Donoghue $M$, Chen $H$, et al. FDA approval summary: pembrolizumab for recurrent locally advanced or metastatic gastric or gastroesophageal junction adnocarcinoma expressing PD-L1. Oncologist 2019;24(1):103-109. DOI: 10.1634/ theoncologist.2018-0221.

18. Higgins JPT, Altman DG, Gøtzsche PC, et al. The Cochrane collaboration's tool for assessing risk of bias in randomised trials. BMJ 2011;343: d5928. DOI: 10.1136/bmj.d5928.

19. Higgins JPT, Thompson SG, Deeks JJ, et al. Measuring inconsistency in meta-analyses. BMJ 2003;327(7414):557-560. DOI: 10.1136/ bmj.327.7414.557.

20. Neyeloff JL, Fuchs SC, Moreira LB. Meta-analyses and Forest plots using a Microsoft Excel spreadsheet: step-by-step guide focusing on descriptive data analysis. BMC Res Notes 2012;5:52. DOI: 10.1186/17560500-5-52.

21. Wallace BC, Schmid CH, Lau J, et al. Meta-Analyst: software for meta-analysis of binary, continuous and diagnostic data. BMC Med Res Methodol 2009;9:80. DOI: 10.1186/1471-2288-9-80.

22. Kudo T, Hamamoto $Y$, Kato K, et al. Nivolumab treatment for oesophageal squamous-cell carcinoma: an open-label, multicentre, phase 2 trial. Lancet Oncol 2017;18(5):631-639. DOI: 10.1016/S14702045(17)30181-X.

23. Doi T, Piha-Paul SA, Jalal SI, et al. Safety and antitumor activity of the anti-programmed death-1 antibody pembrolizumab in patients with advanced esophageal carcinoma. J Clin Oncol 2018;36(1):61-67. DOI: 10.1200/JCO.2017.74.9846.

24. Shitara K, Özgüroğlu M, Bang Y-J, et al. Pembrolizumab versus paclitaxel for previously treated, advanced gastric or gastrooesophageal junction cancer (KEYNOTE-061): a randomised, openlabel, controlled, phase 3 trial. Lancet 2018;392(10142):123-133. DOI: 10.1016/S0140-6736(18)31257-1.

25. Muro K, Chung HC, Shankaran V, et al. Pembrolizumab for patients with PD-L1-positive advanced gastric cancer (KEYNOTE-012): a multicentre, open-label, phase $1 \mathrm{~b}$ trial. Lancet Oncol 2016;17(6):717726. DOI: 10.1016/S1470-2045(16)00175-3.

26. Fuchs CS, Doi T, Jang RW, et al. Safety and efficacy of pembrolizumab monotherapy in patients with previously treated advanced gastric and gastroesophageal junction cancer: phase 2 clinical KEYNOTE-059 trial. JAMA Oncol 2018;4(5):e180013. DOI: 10.1001/ jamaoncol.2018.0013.

27. Janjigian YY, Bendell J, Calvo E, et al. CheckMate-032 study: efficacy and safety of nivolumab and nivolumab plus ipilimumab in patients with metastatic esophagogastric cancer.J Clin Oncol 2018;36(28):2836-2844. DOI: 10.1200/JCO.2017.76.6212.

28. Kang YK, Boku N, Satoh T, et al. Nivolumab in patients with advanced gastric or gastro-oesophageal junction cancer refractory to, or intolerant of, at least two previous chemotherapy regimens (ONO4538-12, ATTRACTION-2): a randomised, double-blind, placebocontrolled, phase 3 trial. Lancet 2017;390(10111):2461-2471. DOI: 10.1016/S0140-6736(17)31827-5.

29. Bang Y-J, Yañez Ruiz E, Van Cutsem E, et al. Phase III, randomised trial of avelumab versus physician's choice of chemotherapy as third-line treatment of patients with advanced gastric or gastro-oesophageal junction cancer: primary analysis of JAVELIN Gastric 300. Ann Oncol 2018;29:2052-2060. DOI: 10.1093/annonc/ mdy264.

30. Harada K, Lopez A, Shanbhag N, et al. Recent advances in the management of gastric adenocarcinoma patients. F1000Research 2018;7:1365. DOI: 10.12688/f1000research.15133.1.

31. Voutsadakis IA. Immune ligands for cytotoxic T lymphocytes (CTLS) in cancer stem cells (CSCS). Front Biosci (Landmark Ed) 2018;23:563-583. DOI: $10.2741 / 4605$. 
32. Voutsadakis IA. Expression and function of immune ligand-receptor pairs in NK cells and cancer stem cells: therapeutic implications. Cell Oncol (Dordr) 2018;41(2):107-121.

33. Panda A, Mehnert JM, Hirshfield KM, et al. Immune activation and benefit from avelumab in EBV-positive gastric cancer. J Natl Cancer Inst 2018;110(3):316-320. DOI: 10.1093/jnci/djx213.

34. Tie $Y, M a X$, Zhu C, et al. Safety and efficacy of nivolumab in the treatment of cancers: a meta-analysis of 27 prospective clinical trials. Int J Cancer 2017;140(4):948-958. DOI: 10.1002/ijc.30501.
35. Taieb J, Moehler M, Boku N, et al. Evolution of checkpoint inhibitors for the treatment of metastatic gastric cancers: current status and future perspectives. Cancer Treat Rev 2018;66:104-113. DOI: 10.1016/j. ctrv.2018.04.004.

36. Stein A, Moehler M, Trojan J, et al. Immuno-oncology in GI tumours: clinical evidence and emerging trials of PD-1/PD-L1 antagonists. Crit Rev Oncol Hematol 2018;130:13-26. DOI: 10.1016/j. critrevonc.2018.07.001. 